Service social

\title{
La famille élargie, incontournable chez les Innus
}

\section{Christiane Guay, Sébastien Grammond et Catherine Delisle-L'Heureux}

Volume 64, numéro 1, 2018

URI : https://id.erudit.org/iderudit/1055893ar

DOI : https://doi.org/10.7202/1055893ar

Aller au sommaire du numéro

Éditeur(s)

École de service social de l’Université Laval

ISSN

1708-1734 (numérique)

Découvrir la revue

Citer cet article

Guay, C., Grammond, S. \& Delisle-L'Heureux, C. (2018). La famille élargie, incontournable chez les Innus. Service social, 64(1), 103-118.

https://doi.org/10.7202/1055893ar

\section{Résumé de l'article}

La famille est au coeur du tissu social des communautés autochtones. Or, contrairement aux sociétés occidentales, les Autochtones ont une conception élargie de la famille. En se fondant sur des récits biographiques de membres de la communauté innue d'Uashat mak Mani-Utenam, le présent texte explore plusieurs facettes du concept de famille élargie. Pour les Innus, la famille élargie donne lieu à un ensemble de devoirs et d'obligations. La cohabitation et la garde coutumière constituent des exemples de formes spécifiques de relations qui découlent du concept de famille élargie. On peut concevoir celle-ci comme un ensemble de cercles concentriques, avec l'enfant au centre, qui définissent des rôles spécifiques. Les allochtones qui interviennent auprès des Innus doivent nécessairement tenir compte de ces spécificités de la famille élargie.
Tous droits réservés ( Service social,
Ce document est protégé par la loi sur le droit d'auteur. L'utilisation des services d’Érudit (y compris la reproduction) est assujettie à sa politique d'utilisation que vous pouvez consulter en ligne.

https://apropos.erudit.org/fr/usagers/politique-dutilisation/ 


\title{
La famille élargie, incontournable chez les Innus
}

\author{
GUAY, Christiane, t.s., Ph.D., Doctorat en sciences sociales appliquées \\ Professeure \\ Département de travail social \\ Université du Québec en Outaouais \\ GRAMMOND, Sébastien, D.Phil, Doctorat en droit \\ Juge \\ Cour fédérale
}

DELISLE-L'HEUREUX, Catherine, professionnelle de recherche

Département de travail social

Université du Québec en Outaouais

\section{RÉSUMÉ}

La famille est au cœur du tissu social des communautés autochtones. Or, contrairement aux sociétés occidentales, les Autochtones ont une conception élargie de la famille. En se fondant sur des récits biographiques de membres de la communauté innue d'Uashat mak Mani-Utenam, le présent texte explore plusieurs facettes du concept de famille élargie. Pour les Innus, la famille élargie donne lieu à un ensemble de devoirs et d'obligations. La cohabitation et la garde coutumière constituent des exemples de formes spécifiques de relations qui découlent du concept de famille élargie. On peut concevoir celle-ci comme un ensemble de cercles concentriques, avec l'enfant au centre, qui définissent des rôles spécifiques. Les allochtones qui interviennent auprès des Innus doivent nécessairement tenir compte de ces spécificités de la famille élargie.

Mots clés : Peuples autochtones, familles, intervention sociale.

\begin{abstract}
The family is at the heart of the social fabric of Indigenous communities. In contrast to Western society, the Indigenous peoples have an extended conception of the family. This paper explores several aspects of the concept of extended family among the Innu of Uashat mak Mani-Utenan, based on the biographical stories of community members. Among the Innu, the extended family gives rise to a array of duties and obligations. Cohabitation and customary care provide examples of specific forms of relationships that flow from the extended family concept. The extended family may be conceptualized as a set of concentric circles, with the child at the centre, which define specific roles. Non-Indigenous persons who intervene with the Innu must necessarily take into account the specific character of the extended family.
\end{abstract}

Keywords: Indigenous people, family, social intervention. 
«La famille est l'élément naturel et fondamental de la société et a droit à la protection de la société et de l'État », affirme l'article 16 de la Déclaration universelle des droits de l'homme. Mais de quelle famille s'agit-il ? La famille nucléaire ${ }^{1}$, cette structure familiale tant valorisée au sein des sociétés occidentales, est-elle la seule qui mérite l'attention? Les peuples autochtones en contexte nordaméricain offrent l'exemple d'un idéal familial fort différent, fondé principalement sur des liens d'interdépendance qui unissent l'ensemble des membres de la famille élargie.

Le présent texte vise à discuter de la conception de la famille au sein de la communauté innue d'Uashat mak Mani-Utenam, située dans le voisinage de la ville de Sept-Îles, sur la Côte-Nord, dans l'est du Québec (Delisle-L'Heureux, 2018). Pour les Innus, en effet, la famille ne se limite pas aux parents et à leurs enfants, mais comprend aussi un réseau élargi de personnes apparentées, des grands-parents aux oncles et tantes, aux cousins et cousines, auxquels s'ajoutent les liens de parenté formés par l'adoption. Souvent, la famille innue tend même à englober la communauté entière. De fait, la facilité avec laquelle les Innus fabriquent de la parenté est telle que l'anthropologue Josée Mailhot (1999) a déjà considéré le caractère extensible de la parenté innue comme une de ses caractéristiques principales.

Les membres de la famille élargie assument des responsabilités déterminées et partagées à l'égard de l'éducation des enfants, si bien qu'elle constitue un filet de sécurité qui assure le bien-être des enfants. Une bonne compréhension du rôle et des responsabilités de la famille élargie est incontournable pour quiconque intervient auprès des familles et des enfants innus. Ignorer ceux-ci risque de mener à des décisions culturellement inadéquates. Or, les enfants autochtones, on le sait, sont surreprésentés au sein des services de protection de la jeunesse (Breton, Dufour et Lavergne, 2012) et cette surreprésentation est particulièrement aiguë dans les cas de négligence. Plusieurs auteurs mettent en cause la prise de décisions par des travailleurs sociaux allochtones à qui les cultures autochtones sont peu familières pour expliquer cette situation (Bertsch et Bidgood, 2010 ; Kline, 1992). Alors que la Loi sur la protection de la jeunesse vient d'être modifiée pour tenir compte du concept de famille élargie en contexte autochtone (art. 4) et que le Code civil reconnaît maintenant l'adoption et la garde coutumière, il devient urgent pour les différents acteurs du système, en particulier les intervenants sociaux, de mieux connaître la famille élargie et le faisceau de responsabilités qui lui est associé.

Le présent texte se fonde principalement sur les résultats d'une recherche portant sur les pratiques éducatives réalisée au sein de la communauté innue d'Uashat mak Mani-Utenam ${ }^{2}$ et de manière plus spécifique sur l'exploration de 10 récits familiaux réalisés auprès de parents et de grands-parents ainsi que de 5 récits communautaires réalisés auprès de différents groupes de la communauté ${ }^{3}$. Dans le cadre de cette recherche, les participants ont été invités à nous parler, entre autres, de leur vision

\footnotetext{
${ }^{1}$ Nous reconnaissons qu'il existe dans les sociétés occidentales une diversité de modèles et de structures familiales. Si nous avons opté pour le concept de famille nucléaire comme point de comparaison pour marquer la spécificité de la famille autochtone, c'est que malgré les transformations actuelles, la famille nucléaire demeure le modèle qui sous-tend, implicitement ou explicitement, la plupart des politiques publiques et de la législation, incluant celles qui portent sur la protection de la jeunesse.

2 II s'agit d'un projet de recherche financé par le CRSH : Pratiques éducatives et de protection de l'enfance : un patrimoine raconté par les Innus d'Uashat mak Mani-Utenam.

3 II s'agit d'un groupe d'intervenants de première ligne, d'un groupe de parents de familles d'accueil, d'un groupe d'intervenants en réadaptation, d'un groupe d'étudiants en techniques d'éducation spécialisée et d'un groupe de parents suivis en protection de la jeunesse.
} 
de la famille innue et de la manière dont on éduque les enfants et en prend soin au sein de la communauté, y compris les enjeux et les défis qui entourent l'éducation des enfants. Nous avons également puisé dans les récits réalisés dans le cadre de trois autres projets de recherche menés au sein de la communauté, lorsque ceux-ci abordaient le sujet de la famille et de l'éducation des enfants ${ }^{4}$. Ainsi que l'énonce le protocole de recherche des Premières Nations du Québec et du Labrador, les Autochtones doivent pouvoir définir les paramètres leur permettant de protéger leurs savoirs tout en assurant la reconnaissance de leur contribution à l'avancement des connaissances (APNQL, 2014). Par conséquent, dans ce projet de recherche, la formule standard de consentement a été modifiée afin de permettre aux participants qui le désiraient de consentir à être identifiés et cités. Cela dit, la confidentialité a été assurée pour tous ceux et celles qui préféraient garder l'anonymat. Dans ces cas, un pseudonyme a été utilisé pour citer leurs propos ${ }^{5}$.

\section{La famille élargie, au cœur des relations sociales innues}

D'entrée de jeu, il importe de préciser que, comme toutes les familles québécoises, les familles des Premières Nations se transforment et s'adaptent au monde contemporain. II n'y aurait donc pas une seule manière de concevoir la famille autochtone (Brant Castellano, 2002 ; Irvine, 2009 ; Lindstrom et al., 2016 ; Muir et Bohr, 2014). Si la plupart des auteurs qui ont écrit sur le sujet nous rappellent l'importance de ne pas réifier le mode de vie des Autochtones, ils concèdent cependant que malgré la diversité régionale, linguistique et sociale, on observe que certaines valeurs, croyances et traditions persistent et qu'elles sont philosophiquement différentes de celles de la société dominante (Guay, 2015 ; Muir et Bohr, 2014).

Cette mise au point effectuée, la famille a toujours été considérée comme l'unité sociale de base de la nation innue (Mailhot, 1999) et malgré les nombreuses transformations qu'elle a connues, la famille s'impose toujours comme une institution centrale autour de laquelle s'organisent les relations sociales, les devoirs et les responsabilités. D'autre part, la famille est pensée le plus souvent non dans son sens restreint de famille nucléaire, telle qu'on la conçoit avant tout en Occident, mais dans son sens plus large qui englobe un vaste réseau de parenté.

II est utile de rappeler qu'il n'y a encore pas si longtemps, les Innus vivaient de manière nomade, parcourant le grand territoire qu'est le Nitassinan à la recherche de poissons et de gibier, en particulier le caribou. De fait, la plus grande partie de l'année, c'était le groupe de chasse familial qui guidait le déplacement quotidien des Innus (Charest, 2001, 2006 ; Lacasse, 2004). Comme le résume bien Lacasse :

II ne semble pas avoir existé, chez les Innus, d'organisation tribale comme chez les peuples Iroquoiens. C'était plutôt la famille (élargie) qui constituait l'unité sociale de base. Quelques familles se réunissaient pour former une bande ou, plus précisément, un groupe de chasse. Les auteurs ont établi une distinction entre la « bande d'hiver », constituée en général d'une à cinq familles pouvant

\footnotetext{
${ }^{4}$ II s'agit de trois projets de recherche dirigés par la chercheure principale : 1) Le territoire comme source de guérison : les séjours thérapeutiques à Uashat mak Mani-Utenam (financé par le FRQSC) ; 2) Les pratiques d'adoption coutumière chez les autochtones: la perspective des Innus d'Uashat mak Mani-Utenam (mené dans le cadre d'un partenariat de recherche dirigé par le professeur Ghislain Otis de l'Université d'Ottawa : État et cultures juridiques autochtones : un droit en quête de légitimité [Legitimus] et financé par le $\mathrm{CRSH}$ ) ; 3) La rencontre des savoirs à Uashat mak Mani-Utenam : regards des intervenants sociaux innus sur leur pratique, un projet sur la pratique des intervenants sociaux (thèse de doctorat).

${ }^{5}$ Certains passages peuvent aussi avoir été omis des citations afin d'assurer l'anonymat de ces participants.
} 
compter jusqu'à 75 personnes et la bande d'été qui rassemblait, sur les bords d'un lac ou à la côte, deux ou trois bandes d'hiver, soit 130 à 200 personnes (Lacasse, 2004, p. 30).

La sédentarisation des Innus, au cours du $X X^{e}$ siècle, a transformé considérablement leur mode de vie (Charest, 2001, 2006). Charest (2001) souligne d'ailleurs que pour la grande majorité des Innus, la sédentarisation est un phénomène très récent ${ }^{6}$. Malgré des changements en profondeur sur le plan technologique, économique, politique, social et spirituel, la famille élargie n'a pas perdu son importance. En effet, si la communauté agit comme nouvelle sphère de socialisation, des auteurs ont conclu que les réseaux de socialité s'organisent toujours autour des familles. Par exemple, Mailhot, à propos de la communauté innue de Sheshatshit, a observé que « les individus ne circulent guère qu'à l'intérieur de leur cercle de parenté » (Mailhot, 1999, p. 60). Gentelet, Bissonnette et Rocher, quant à eux, ont bien vu qu'en contexte sédentaire, les Innus ne se représentent plus le cercle des relations de la même manière, alors que « le cercle des relations, autrefois circonscrit à la famille, s'est élargi à la communauté [...] » (Gentelet, Bissonnette et Rocher, 2005, p. 25). Or, malgré l'élargissement du cercle des relations, la famille demeure toujours au cœur du tissu social des communautés et de la nation innue, puisque c'est l'interdépendance entre les membres qui composent ce cercle de relations qui permet de soutenir l'ensemble de la communauté (Irvine, 2009). Ici, il n'est pas inutile de rappeler que la structure familiale autochtone :

[...] commence avec les Aînés de la famille et s'étend non seulement verticalement pour englober leurs enfants et petits-enfants, mais aussi latéralement, de manière à englober tous les frères et sœurs adultes, les cousins, leur conjoint et leurs enfants. Même les amis proches sont des membres d'honneur de la famille et prennent part de façon routinière aux activités familiales. L'entraide et les relations d'échange entre les membres des familles autochtones tissent des liens solides entre eux et forment une unité intime, soli[d]aire qui profite à toutes les générations et à tous ses membres (Thomas et Learoyd, 1990, dans Irvine, 2009, p. 15).

Les récits innus que nous avons recueillis attestent de réalités similaires. En effet, plusieurs participants nous ont parlé de leur attachement à leurs relations familiales, qui s'étendent bien au-delà de la famille nucléaire. En témoigne ce parent de famille d'accueil, pour qui : « La famille innue, c'est d'abord ma famille immédiate. C'est la famille à l'intérieur de notre famille : mes frères et sœurs, mes tantes, les enfants, les petits-enfants. " D'autres n'hésitent pas à présenter la communauté dans son ensemble comme une extension de la famille :

Nous vivons dans une petite communauté et ça crée des liens. Par exemple, mon voisin, même s'il ne fait pas partie de ma famille, il finit par le devenir parce que j'ai créé des liens avec lui. C'est ça une communauté (Shaunien).

La communauté fait aussi partie de la famille. Les enfants n'apprennent pas juste à la maison. En vivant dans la communauté, ils apprennent des valeurs. C'est une force (Paroles de familles d'accueil).

Par ailleurs, la famille occupe une place centrale dans la vie quotidienne des Innus, en ce sens que c'est à partir de sa famille que l'Innu tisse ses relations, appartient à sa communauté, reçoit et transmet des valeurs, notamment celle de l'entraide. Comme l'expliquent ces participantes :

\footnotetext{
${ }^{6}$ La réserve d'Uashat a été créée en 1904 et celle de Mani-Utenam en 1949.
} 
La famille innue est une famille unie, tissée serrée, qui se respecte. Une famille ouverte aux valeurs culturelles traditionnelles et aux valeurs du monde d'aujourd'hui. Une famille qui comprend l'importance de transmettre l'histoire et les connaissances aux générations futures (Shipiss).

Pour moi, dans une famille, les gens peuvent se parler, s'exprimer et ils se respectent mutuellement. [...] les membres d'une famille sont là pour s'entraider lorsque ça ne va pas. La famille est aussi là pour célébrer les réussites et les bons moments (Bernadette).

L'entraide est sans nul doute la valeur que les participants ont le plus souvent mentionnée dans leurs récits. Selon Maria, "nous avons toujours gardé cette valeur de l'entraide », alors que Line affirme que «l'entraide, c'est notre grande force ». De nombreux exemples concrets d'entraide nous ont été donnés, qu'il serait trop long d'énumérer ici.

En somme, dans une perspective d'appréciation des responsabilités partagées en matière de prise en charge et d'éducation des enfants par la famille élargie et la communauté, les constats dont nous venons de faire état sont d'une importance capitale : la famille élargie et la communauté - entendue comme son extension - constituent le véhicule de la transmission culturelle, notamment des valeurs, et le socle sur lequel repose le développement de l'identité chez l'enfant. Pour ce dernier, sa famille et sa communauté forment un filet de sécurité où il peut découvrir sa place dans le monde, connaître ses droits et ses responsabilités et acquérir les conditions nécessaires à son épanouissement. L'enfant a besoin de sa famille et de sa communauté ; réciproquement, ces dernières ont aussi besoin des enfants, qui forment la vitalité des familles et l'avenir de la communauté. Comme le dit bien Carine : « Les enfants représentent la vie, l'avenir, l'espoir. Ce sont eux qui feront en sorte que notre culture se perpétuera. »

\section{Des devoirs et responsabilités spécifiques à l'égard des enfants}

Le caractère distinctif de la famille élargie innue est l'ensemble de devoirs et de responsabilités qui lient les membres les uns envers les autres. En effet, si les Innus qui se sont entretenus avec nous utilisent les termes de parenté que nous connaissons tous (frère, tante, grand-mère, etc.), ils se reconnaissent des responsabilités particulières envers les membres de leur famille élargie. Ce sont donc ces responsabilités qui seraient au cœur de la conception innue de la famille, davantage qu'un système original de parenté.

Cette situation peut être comparée avec la famille nucléaire québécoise. Selon le Code civil, seuls les parents ont une obligation alimentaire envers les enfants, et vice versa ${ }^{7}$. Or, aucune restriction de ce genre n'existe au sein de la famille élargie innue. Deux exemples, la cohabitation et la garde coutumière, permettent d'illustrer l'étendue de ces responsabilités.

La cohabitation est une pratique largement répandue à Uashat mak Mani-Utenam. Un premier facteur à considérer est certainement la crise du logement. D'ailleurs, certains participants nous ont parlé du manque de logements pour expliquer pourquoi plusieurs membres d'une famille élargie vivent sous le même toit (Line) ainsi que des problèmes familiaux qui sont parfois engendrés par le manque de logement (Shushep et Kananine). Cela étant dit, la crise du logement est loin de fournir à elle seule une explication suffisante au phénomène de cohabitation. En effet, la cohabitation semble également découler de pratiques sociales et culturelles distinctes qui sont animées par les valeurs d'entraide et

\footnotetext{
${ }^{7}$ L'obligation alimentaire entre grands-parents et petits-enfants a été abolie en 1997.
} 
de partage. Pour de nombreux Innus, le fait d'habiter à plusieurs sous un même toit est souvent le résultat d'un choix conscient, visant à s'entraider, à prendre soin d'un membre de sa famille, plutôt que le résultat d'une situation non désirée. Julia et Anna en donnent un exemple frappant :

Une fois mes études terminées, j'aurais pu partir en appartement, mais [...], mon grand-père est décédé. Le plus jeune de mes oncles et moi-même sommes restés avec ma grand-mère. À partir de ce moment-là, il était hors de question que je laisse ma grand-mère toute seule, de même que je ne l'aurais jamais emmenée en résidence. Je pouvais aller en appartement ou commencer à construire ma maison, mais j'ai décidé de rester avec elle (Julia).

Un an après sa mort [grand-père], ma tante et mes oncles sont également venus s'installer ici, avec ma sœur et leurs enfants. Nous avons toujours vécu ensemble. Trois familles ensemble. Nous nous entraidions en ce qui a trait à la nourriture et à la chasse. C'est comme ça que nous avons grandi et appris [...] (Anna).

Pour les jeunes parents, la cohabitation est aussi un arrangement avantageux qui peut favoriser l'acquisition de compétences parentales. II ne faut pas oublier que chez les Innus, il n'est pas rare qu'une mère habite encore chez ses parents au moment où elle donne naissance à son premier enfant. Par exemple, Carine raconte: "Quand je suis tombée enceinte, nous habitions dans la maison de mes grands-parents, avec ma mère et ma tante jusqu'à ce que ma maison soit construite. Elles m'ont appris à m'occuper du bébé. » Elle se rappelle même son jeune âge, dans la maison de ses grands-parents, lorsque ceux-ci pouvaient prendre le relais lorsque sa mère était absente :

Par exemple, quand une fille-mère tombe enceinte, elle n'a pas sa propre maison : elle vit chez ses parents. L'enfant va naître dans un milieu qui lui ressemble et se sentir en sécurité. Même si ma mère travaillait en dehors de la communauté, je n'étais jamais toute seule chez mes grands-parents : il y avait ma grand-mère, mon grand-père, mes tantes et mes oncles. J'ai donc vécu dans la sécurité et la stabilité (Carine).

Dans les faits, il est tout à fait normal et même fréquent pour les jeunes familles ou jeunes mères de vivre sous le même toit que leurs parents ou grands-parents. II y a donc plusieurs personnes qui gravitent autour de l'enfant et qui, à des degrés divers, vont s'en occuper. Les propos suivants en fournissent une illustration.

Ma famille est pas mal élargie et c'est très harmonieux. J'ai une sœur qui s'occupe d'une de mes filles (le bébé), ma mère s'occupe de ma plus vieille (qui vient d'avoir un bébé) et ma petite-fille et mon frère habitent chez elle avec ses deux enfants. S'il manque quoi que ce soit à ma fille (aînée qui vient d'avoir un enfant) elle se tournera vers sa grand-mère si je ne peux combler son besoin (Alanis).

En somme, bien qu'à certains égards la cohabitation découle du manque de logements, les résultats de notre recherche mettent en lumière le fait que le mode de vie des familles innues, comme celui de bon nombre de familles des Premières Nations, est avant tout ancré dans une structure de parenté complexe fondée sur des valeurs d'entraide et de respect qui dictent un ensemble d'obligations et de responsabilités entre les membres de la famille élargie, plus spécifiquement les grands-parents envers les petits-enfants (Guay et Grammond, 2012 ; Lindstrom et al., 2016 ; Muir et Bohr, 2014). Dans bien des cas, on peut dire que la cohabitation, non seulement permet de tisser des liens solides et de réciprocité entre les membres de la famille élargie, mais agit également comme facteur de protection à l'égard des enfants. Dans ce contexte, l'éducation et la protection des enfants innus sont 
des responsabilités qui sont partagées par l'ensemble des membres de la famille et pas seulement les parents biologiques.

Une autre pratique, celle du ne kupaniem/ne kupanishkuem, que l'on peut traduire en français par « un ou une enfant que l'on garde temporairement» et qui renvoie à une pratique d'adoption coutumière ou plus exactement de garde coutumière ${ }^{8}$, illustre aussi les responsabilités des membres de la famille élargie. Cette pratique, présente au sein de plusieurs Premières Nations, consiste à transférer la garde d'un enfant à un membre de la famille élargie, souvent aux grands-parents, parfois aux oncles et tantes, lorsque les parents d'origine sont incapables d'en prendre soin, quelle qu'en soit la raison (Lindstrom et al., 2016 ; di Tomasso et de Finney, 2015). Nos recherches au sein de la communauté d'Uashat mak Mani-Utenam ont démontré que le transfert est réalisé par entente expresse ou tacite entre les personnes concernées. Celle-ci n'implique pas le recours à une personne en situation d'autorité ou à une procédure formelle ou écrite. Ce transfert est en principe temporaire et réversible. II n'efface pas la filiation d'origine, ce qui le distingue de l'adoption en droit québécois. L'enfant connaît ses parents d'origine. On l'encourage à maintenir des contacts avec ceux-ci et même à retourner vivre chez eux au début de l'adolescence (Basile, Rock et Rock, 1992 ; Grammond et Guay, 2016). Lorsque la situation perdure, elle peut conduire à l'apparition d'un nouveau lien de filiation, en plus du lien d'origine. Bien que cette pratique tire son origine du mode de vie traditionnel et des déplacements saisonniers que celui-ci comporte (Lanari, 1973) ${ }^{9}$, elle est encore très fréquente au sein de la société innue contemporaine.

La pratique du ne kupaniem/ne kupanishkuem est une manière de maintenir en vie la valeur de l'entraide. Anna, par exemple, affirme: « Je voulais aider mes enfants en élevant mes petits-enfants et arrière-petits-enfants. » En ce sens, cette pratique participe des responsabilités qui sont associées à la famille élargie.

De cette manière, la garde coutumière joue un rôle essentiel dans le bien-être et la protection des enfants au sein de la communauté. Elle permet à l'enfant de créer et de maintenir des liens significatifs avec un ensemble de personnes, non seulement avec les membres de la famille élargie de sa famille adoptive, mais également avec ceux de sa famille d'origine. L'enfant est assuré ainsi d'un vaste réseau d'appartenance qui fonctionne ni plus ni moins comme un filet de sécurité pour l'enfant (Grammond et Guay, 2016). Lors d'une rencontre de groupe, une intervenante de première ligne a bien décrit les bénéfices qui découlent de l'extension de son réseau familial :

Dans ma jeunesse, plusieurs personnes prenaient soin de moi, pas seulement mes parents. Grâce à cela, j'ai pu créer des liens avec mes tantes, mes oncles, mes cousins. Aujourd'hui, je suis entourée de bonnes personnes, d'êtres de confiance. Quand j'ai besoin de support, je peux aller les voir. J'ai pu élargir mes liens au-delà de la petite famille (Paroles d'intervenants de première ligne).

Historiquement, la garde coutumière avait pour fonction d'assurer la survie sur le territoire en assurant aux Autochtones un vaste réseau de connaissances apparentées. Elle avait aussi pour rôle de pallier

\footnotetext{
${ }^{8}$ Les concepts d'adoption et de garde coutumière sont des concepts qui sont souvent critiqués puisqu'ils ne trouvent pas leur équivalent dans la plupart des langues autochtones et parce qu'ils ne rendent pas compte de la richesse et de la diversité des valeurs et des pratiques entourant les soins et le bien-être des enfants autochtones. C'est la raison pour laquelle nous optons pour le terme utilisé par les Innus eux-mêmes.

9 Selon Lanari (1973), le caractère temporaire de l'adoption en contexte innu s'explique notamment par le fait que dans le contexte du nomadisme, l'adoption d'un enfant par une famille n'avait lieu souvent que pour une saison, autrement dit, le temps d'une remontée dans le territoire en période hivernale.
} 
l'infertilité de certains couples et de combler les besoins de certaines familles (di Tomasso et de Finney, 2015 ; Mailhot, 1999). Aujourd'hui, la garde coutumière n'a plus de fonction liée à la survie sur le territoire, cependant, les résultats de notre étude (Grammond et Guay, 2016) font ressortir qu'elle est devenue un moyen singulier de prendre soin et de protéger les enfants tout en respectant l'intérêt de l'enfant.

En effet, les parents confient la garde de leur enfant à une autre personne pour différentes raisons : parce qu'ils doivent quitter temporairement la communauté pour des raisons liées aux études ou à l'emploi, en raison de leur jeune âge (grossesse à l'adolescence), ou parce qu'ils sont aux prises avec des problèmes personnels, comme la consommation d'alcool ou de drogue :

J'ai vécu avec ma mère jusqu'à l'âge de cinq ans et ensuite, comme ma mère consommait, ce sont mes grands-parents qui ont pris soin de moi. J'ai grandi avec eux (Paroles d'étudiants en techniques d'éducation spécialisée).

Moi aussi, j'allais chez ma grand-mère lorsque ma mère consommait. Je m’y sentais en sécurité. Elle s'occupait de moi (Paroles d'étudiants en techniques d'éducation spécialisée).

Dans ces cas, ce sont bien souvent les parents qui font eux-mêmes le constat de leur incapacité à s'occuper convenablement de leur enfant (Grammond et Guay, 2016). Une participante décrit cette situation comme suit :

[P]arfois c'est justement parce que ses parents sont conscients qu'ils ne peuvent pas s'occuper de lui adéquatement qu'ils choisissent de le placer chez des gens qui seront en mesure de le faire. Une mère ne peut pas donner ce qu'elle n'a pas. Parfois, elle n'a pas la santé ou l'équilibre psychologique pour élever l'enfant adéquatement. C'est un geste d'amour et d'humilité d'avouer être incapable de s'occuper de son enfant. C'est souvent parce que la mère n'est pas en mesure de s'occuper d'ellemême qu'elle pose ce geste (Carine).

Bref, le fait de garder ou de prendre soin d'un enfant qui n'est pas biologiquement le nôtre est une pratique qui est, aujourd'hui encore, très fréquente à Uashat mak Mani-Utenam. II n'est pas atypique qu'un enfant vive plusieurs changements de garde durant sa vie, sans que cela cause une perte de stabilité. Au contraire, il s'agit d'une force que d'avoir plusieurs liens de parenté. Certes, la pratique s'est transformée au fil du temps, mais elle préserve les mêmes caractéristiques fondamentales : une souplesse dans la pratique, une réponse adaptée aux besoins du moment qui peut changer selon les besoins de l'enfant et des parents. Elle est, comme l'a bien dit Lavallée (2013), informelle et consensuelle, ancrée dans l'idée que d'autres personnes que les parents biologiques peuvent très bien prendre soin d'un enfant.

\section{Le cercle des relations}

Si le concept de famille élargie a pour effet d'attribuer à un grand nombre de personnes des responsabilités à l'égard d'un enfant, il ne s'ensuit pas que ces responsabilités sont indifférenciées. Les Innus ont malgré tout une représentation des rôles assumés par les différents membres de la famille. On peut donc concevoir celle-ci comme un ensemble de cercles concentriques. L'enfant se retrouve au milieu; le premier cercle est constitué de ses père et mère ; un second cercle de ses frères et sœurs, ses grands-parents, ses oncles et ses tantes, puis un troisième des parents plus éloignés. Enfin, la communauté, la nation innue et toutes les Premières Nations constituent les cercles 
extérieurs. Le positionnement de ces divers individus au sein de ce cercle n'est pas rigide. II peut être réaménagé, par exemple dans une situation de garde coutumière. Le cercle des relations trouve sa source dans « la vision relationnelle du monde des cultures autochtones, selon laquelle tous les gens et les choses sont interreliés, et où la notion de relation constitue la pierre angulaire de la communauté » (Cajet, 2000, dans Lindstrom et al., 2016, p. 13, notre traduction).

Ainsi, les Innus reconnaissent à la mère et au père le rôle de premiers pourvoyeurs de soins. Une étudiante en techniques d'éducation spécialisée a affirmé : «C'est le premier rôle de la mère de protéger ses enfants. C'est notre responsabilité comme parents et comme grands-parents ensuite » (Paroles d'étudiants en techniques d'éducation spécialisée). Pour plusieurs, les responsabilités de la mère et du père sont différenciées. D'un côté, la mère serait responsable des soins :

Dans la culture innue, le rôle des mamans est de prendre soin des enfants et de les protéger. [...] Comme les mamans restent à la maison avec les enfants, leur lien est plus fort, c'est normal (Kateri).

La maman innue se soucie de la sécurité des enfants, c'est une maman-infirmière, qui anticipe les difficultés. Elle joue un rôle préventif. Elle se préoccupe de la santé physique des enfants (Shushep et Kananine).

Sans nier le rôle du père dans les soins à donner aux enfants, certains participants considèrent que le rôle du père est d'une nature plus ludique :

Les papas sont là pour jouer et travailler (Kateri).

Le père est plus joueur et plus actif (Shushep et Kananine).

Mon chum est plus «gaga » que moi : il joue avec les enfants, il les gâte [...] Son rôle comme père est plutôt de nous protéger et de nous apporter une sécurité financière (Léa).

Certains cependant attribuent aux pères un rôle plus marqué. Par exemple, Shushep affirme que ceux-ci « ont le défi d'être présents et d'accompagner leurs enfants ». Si le rôle du parent consiste à protéger les enfants et à subvenir à leurs besoins de base, d'autres, comme Annie, précisent qu'il incombe également aux parents d'assurer l'apprentissage de la culture et de l'identité : « Je dirais que le rôle du parent consiste à donner les outils aux enfants pour qu'ils aient de bonnes bases, de bonnes valeurs ainsi que des connaissances sur leur culture et leur identité. Le but est que les générations futures soient solides et fassent la différence dans les communautés »(Annie).

D'ailleurs, sans nécessairement faire de distinction entre les rôles paternel et maternel, certaines mères reconnaissent à leur conjoint des compétences particulières dans le domaine de l'éducation culturelle :

Dans notre famille, le père joue le même rôle que la mère, bien que mon conjoint soit plus habile que moi en ce qui concerne l'éducation culturelle. Par exemple, c'est lui qui montrera à nos enfants à bucher du bois. Nous nous efforçons de jouer notre rôle dans le respect de l'autre. II ne s'ingère pas dans mon intervention et vice versa (Shipiss).

Parfois, il lui arrivait de se sentir inutile à la maison, alors je le rassurais en lui disant qu'il servait à quelque chose parce que grâce à lui nous pouvions manger de la « viande de bois ». [...] Je lui disais aussi que grâce à lui, les enfants savaient ce que c'était que d'être un vrai Innu et d'aller dans le bois. Pour moi, c'était très important que les enfants apprennent ça. Les enfants allaient tous avec lui dans le bois (Line). 
Ces commentaires témoignent de pratiques assez genrées en matière de parentalité, ce qui peut s'expliquer en partie par le fait que l'éducation des enfants, dans le territoire, suivait traditionnellement une certaine division sexuelle des tâches. Dans tous les cas, à l'instar de l'étude de Boucher (2005) à propos de la transmission des savoirs chez les Atikamekw, retenons surtout la contribution centrale des parents à la transmission des savoirs auprès de leurs enfants.

Les grands-parents jouent aussi incontestablement un rôle central dans l'éducation des enfants. Bon nombre de témoignages traduisent le lien particulièrement fort qui unit les grands-parents et leurs petits-enfants. En voici quelques exemples :

Ma grand-mère paternelle était très présente, malgré tous les enfants et les petits-enfants qu'elle a eus. Nous étions tout le temps avec elle, notre lien était très fort (Paroles d'étudiants en techniques d'éducation spécialisée).

On dirait que les enfants et les aînés ont besoin les uns des autres (Line).

Les grands-parents ont aussi des responsabilités envers leurs petits-enfants. Lors d'une rencontre de groupe, des intervenants de première ligne nous ont dit : "Nous nous sentons toujours responsables de ce qui arrive à nos enfants et petits-enfants » et "lorsqu'on est grand-mère ou grand-père, on subvient en partie aux besoins de ses petits-enfants". Certaines responsabilités spécifiques incombent aux grands-parents. Ainsi, on ne se surprendra pas que la transmission de la culture relève de leur domaine privilégié, comme l'indique cet intervenant : "Les aînés jouent également un rôle de protection et de transmission de la culture et des connaissances " (Paroles d'étudiants en techniques d'éducation spécialisée). Et cela, non seulement en ce qui a trait aux habiletés liées à la vie en forêt, mais aussi en ce concerne l'histoire orale, comme nous le rappelle Kateri :

Ma grand-mère a joué et joue encore un rôle important dans ma vie. Je vais régulièrement lui rendre visite avec mon garçon. [...] Elle joue un rôle de réconfort. Elle a eu 14 enfants et elle a vécu dans le bois. J'aime qu'elle raconte ses histoires. Ici, elle ne parle pas beaucoup, mais dans le bois, elle s'exprime davantage. Une fois, nous sommes allés sur les lieux de sa naissance et toute la nuit, elle nous a raconté tout ce qui lui venait à l'esprit, elle n'arrêtait pas.

Le rôle des grands-parents est aussi d'appuyer les parents dans le soin et l'éducation de leurs enfants. Cette responsabilité des grands-parents est particulièrement pertinente dans les cas où les parents cohabitent avec les grands-parents. C'est notamment ce qu'évoque Mani :

Ma grand-mère m'a appris beaucoup de choses. Avant, je criais beaucoup. [...] Quand je criais, elle m'entendait jusque chez elle. Elle venait chez moi et me disait d'arrêter de crier, d'habiller mes enfants et de les emmener chez elle. Je le faisais et nous restions une semaine là-bas. [...] Je disais toujours à ma grand-mère que j'avais mal à la tête. [...] Une des fois où nous sommes allés passer du temps chez elle, après avoir couché les enfants, je suis allée retrouver ma grand-mère au salon et elle m'a demandé si j'avais mal à la tête. Je lui ai répondu non. Elle m'a dit que c'est parce que je n'avais pas crié. [...] À partir de ce moment-là, j'ai arrêté de crier et j'ai donné la chance à mes enfants de s'amuser en toute liberté.

Plusieurs grands-parents voient également la garde coutumière comme une de leurs responsabilités. Une intervenante de première ligne a affirmé : "Moi aussi, je garderais mes petits-enfants si leurs parents étaient en difficulté. » Un autre récit témoigne des efforts d'une grand-mère pour se faire confier la garde de ses petits-enfants placés en famille d'accueil (Saulnia). Une intervenante de 
première ligne va jusqu'à dire qu'elle renoncerait à son emploi si cela était nécessaire pour lui permettre d'assumer la garde de ses petits-enfants et d'éviter qu'ils soient placés en famille d'accueil non autochtone (Paroles d'intervenants de première ligne). En somme, ces récits confirment ce que plusieurs auteurs ont dit sur le rôle majeur joué par les grands-parents dans l'éducation des enfants, sur le plan de la transmission de la culture, des soins et de l'affection à donner aux enfants, ou encore de la prise en charge complète ou partielle des responsabilités parentales (Boucher, 2005 ; Brunschwig, 2015 ; Lavallée, 2013).

D'autres membres de la famille élargie, notamment les oncles et les tantes, peuvent aussi apporter leur soutien à l'éducation d'un enfant. Par exemple, Kateri nous parle de l'aide qu'elle a reçue de sa belle-sœur et de sa tante :

Nous avons aussi beaucoup de soutien de sa famille parce que tous ses frères et ses sœurs habitent à côté de chez nous, à Uashat. J'ai toujours bénéficié d'un bon soutien quand j'ai eu besoin d'aide. Par exemple, quand j'ai commencé à travailler, mon bébé avait quatre mois, et sa tante s'occupait de lui. Ça ne me dérange pas que quelqu'un d'autre s'occupe de lui, mais je ne veux pas qu'il soit à la garderie trop longtemps.

Ma mère vient souvent chez nous, mais c'est ma tante qui m'aide le plus. Elle a été très présente après la naissance du bébé. Elle venait faire mon ménage, s'occuper de mon bébé et m'aider pour l'allaitement. Elle a été une aide très précieuse au début, surtout pour la nuit. Lorsque mon chum partait travailler à l'extérieur, elle venait dormir à la maison.

De façon générale, plusieurs participants nous ont parlé de l'entraide qui règne dans leur vie familiale. Les membres de la famille élargie entourent les enfants, en prennent soin et tissent des liens significatifs avec eux. D'une certaine manière, on peut dire que toutes les personnes de la parenté contribuent à créer un cercle de soutien qui prend forme dès la naissance d'un enfant et qui se maintient tout au long de sa vie.

La famille élargie prend une extension telle qu'elle englobe ultimement la communauté tout entière. Certains n'hésitent pas à s'approprier le proverbe bien connu selon lequel «ça prend toute une communauté pour élever un enfant "(Paroles de parents). La communauté serait un lieu où l'on apprend les valeurs (Paroles de familles d'accueil). Les activités comme le hockey ou la garderie favoriseraient d'ailleurs le développement de l'esprit communautaire (Paroles d'intervenants de première ligne).

La mobilité des enfants dans leur quotidien est une illustration du rôle de la communauté dans la prise en charge des enfants. Dans les maisons, les enfants entrent et sortent, de sorte qu'il n'y a pas vraiment de coupure entre la vie familiale et la vie communautaire. À titre d'illustration, les parents ne préparent pas seulement le repas pour leurs propres enfants, mais aussi pour tous ceux qui sont présents dans leur maison. Par exemple :

Ma maison est quasiment une maison communautaire d'enfants. Ils entrent et ils sortent sans arrêt, ils entrent par la porte, ils sortent par le patio. On ne voit jamais cela chez les Blancs. J'achète de la bouffe en fonction des enfants qui vont venir. Nous ne sommes que deux et pourtant, nous achetons beaucoup de nourriture (Paroles d'intervenants de première ligne).

On peut aussi prendre ma maison comme exemple. Ici, il n'y a pas vraiment de terrain privé. J'ai un trampoline et des balançoires et quasiment tous les enfants du village viennent jouer dans ma cour. 
Je connais les parents des enfants ; ils savent qu'ils sont là. [...] Accueillir les gens à la maison, c'est une habitude que mes parents m'ont laissée (Saulnia).

En réalité, garder sa porte ouverte aux enfants de la communauté est un corollaire du fait que chez les Innus, la maison n'est pas considérée comme un espace privé de la même manière que dans la société québécoise. Le fait d'accueillir d'autres membres de la communauté est valorisé et certains participants évoquent avec admiration le souvenir de leurs grands-parents dont la maison était ouverte à tous, notamment à ceux qui étaient dans le besoin. Ainsi, ces deux participantes parlent de leur grand-mère :

Mes grands-parents étaient de bonnes personnes. Ma grand-mère, c'était la bonté incarnée! Sa porte n'était jamais barrée et elle l'ouvrait à quiconque se promenait dehors et avait faim. Tout le monde était bienvenu chez elle (Rosanne).

Elle [ma grand-mère] accueillait chez elle les personnes qui n'avaient nulle part où aller, parce qu'ils venaient de l'extérieur ou parce qu'ils buvaient, et elle leur donnait à manger. Elle démontrait beaucoup d'empathie et de respect de l'autre. Elle faisait attention aux gens parce qu'elle savait qu'ils avaient de la misère. Elle les accueillait à bras ouverts, leur disant que le frigidaire serait toujours plein pour eux (Paroles d'étudiants en techniques d'éducation spécialisée).

Par ailleurs, la circulation quotidienne des enfants dans la communauté fait que la responsabilité de surveiller les enfants appartient à tous les membres de la communauté. S'il arrive un incident, les enfants savent qu'ils peuvent aller chercher de l'aide chez un voisin ou une personne de l'entourage. Une étudiante en techniques d'éducation spécialisée a dit qu'elle acceptait de laisser son enfant se promener seul dans la communauté : "Je n'ai pas peur parce que nous sommes une petite communauté et que les voisins veillent aussi sur elle. " De son côté, une intervenante de première ligne affirme : " II y aura toujours un voisin qui va être au courant et qui va protéger l'enfant. Les gens vont se mobiliser autour de l'enfant qui a besoin de protection. "Par exemple, Shaunien raconte avoir, en compagnie d'une collègue non autochtone, secouru une fillette qui avait fait une chute de vélo :

Hier, il y avait deux petites filles qui faisaient de la bicyclette pas très loin d'ici. Elles devaient avoir environ 3 ans. L'une d'elles est tombée. [...] J'ai expliqué à ma collègue que les enfants dans la communauté, ils nous appartiennent tous. Nous venions d'agir de la même façon que nous l'aurions fait si l'enfant avait été le nôtre. Les enfants sont en sécurité ici car tout le monde les surveille.

En somme, le rôle de la communauté dans la prise en charge des enfants ne fait aucun doute et s'inscrit en continuité avec le soin et la protection des enfants qu'assure la famille élargie. En ce sens, les responsabilités à l'égard des enfants sont partagées. Et s'il existe certaines attentes vis-à-vis des différentes figures parentales, selon qu'il s'agit des parents biologiques, des grands-parents, des membres de la famille élargie ou des membres de la communauté, il n'en demeure pas moins que la division des rôles familiaux est plus fluide chez les Innus. Dans tous les cas, ces récits démontrent amplement l'importance de reconnaître les rôles joués par les membres de la famille élargie - ainsi que par les membres de la communauté - quand vient le temps d'intervenir auprès des familles et des enfants autochtones et plus spécifiquement dans le domaine de la protection de la jeunesse. 


\section{Mieux reconnaître la famille élargie}

Ne pas tenir compte du concept de famille élargie, ou, plus précisément, ne pas considérer la famille élargie comme faisant partie intégrante de «la famille», peut donner lieu à des décisions culturellement inadaptées, voire à la judiciarisation de la situation d'un enfant pour des motifs artificiels (Guay, 2015 ; Irvine, 2009 ; Muir et Bohr, 2014). Une décision récente de la Cour du Québec en donne un exemple ${ }^{10}$. II s'agit du cas d'un enfant qui, dès sa naissance, a été pris en charge par sa grand-tante maternelle. Sa mère est décédée par la suite, alors que le père n'est pas inscrit à l'acte de naissance et s'est rarement impliqué dans la vie de l'enfant. II en résulterait, selon l'intervenante de la DPJ, dont le rapport est cité dans le jugement, " une situation de responsabilité parentale non assumée ». Pourtant, du même souffle, elle reconnaît que la grand-tante subvient à tous les besoins de l'enfant. La judiciarisation de ce dossier est donc fondée sur une évaluation de la situation de l'enfant qui fait totalement abstraction des responsabilités de la famille élargie, dans ce cas-ci, de la garde coutumière de l'enfant par sa grand-tante maternelle. Paradoxalement, le juge place l'enfant auprès de sa grand-tante, qui souhaite l'adopter légalement. Ultimement, la famille élargie est reconnue, mais au prix de contorsions intellectuelles majeures et d'une visite au tribunal.

Or, de récentes modifications législatives permettent d'entrevoir un dialogue plus respectueux entre les conceptions occidentale et innue de la famille. En juin 2017, le Code civil a été modifié pour permettre la reconnaissance de l'adoption et de la garde coutumière (art. 199.10 et 543.1). Selon les nouvelles dispositions du Code civil, une autorité désignée par chaque communauté peut émettre un certificat qui a pour effet soit de transférer la tutelle de l'enfant (y compris l'autorité parentale à son égard) aux membres de la famille élargie qui en prennent soin, soit de consacrer un véritable changement des liens de filiation, dans le cas d'une adoption. De plus, une modification corrélative à la Loi sur la protection de la jeunesse (l'article 71.3.1) ${ }^{11}$ oblige le directeur de la protection de la jeunesse (DPJ) à envisager la tutelle ou l'adoption coutumière autochtone à titre de mesure susceptible d'assurer l'intérêt de l'enfant.

Par ailleurs, les modifications apportées à la Loi sur la protection de la jeunesse en octobre 2017 tiennent compte du concept de famille élargie. Ainsi, un nouvel alinéa a été ajouté à l'article 4 de cette loi, qui vise les cas où un enfant doit être placé en dehors de sa famille (nucléaire) :

Une décision prise en application du deuxième ou du troisième alinéa à l'égard d'un enfant autochtone doit tendre à confier cet enfant à un milieu de vie substitut en mesure de préserver son identité culturelle, en privilégiant un membre de la famille élargie, de la communauté ou de la nation de l'enfant.

Le législateur québécois témoigne donc d'une certaine ouverture aux réalités de la famille innue. II reste à savoir si ces modifications récentes de la Loi sur la protection de la jeunesse, une fois entrées en vigueur, se traduiront par des changements concrets dans les pratiques. En particulier, on peut se demander si des déclarations de principe telles que l'article que nous venons de citer peuvent conduire à un changement de mentalité chez les intervenants chargés d'appliquer la loi sur le terrain. Des mesures de formation et de sensibilisation seront sans doute nécessaires. II faudra faire preuve de vigilance et mesurer les effets concrets de ces déclarations. En dernière analyse, c'est sans doute

10 Protection de la jeunesse - 163249, 2016 QCCQ 7746, en ligne : http://canlii.ca/t/gswxx.

${ }^{11}$ Cette modification a été adopté, mais n'est pas encore en vigueur 
le développement de systèmes autochtones de protection de la jeunesse, indépendants du système québécois, qui constitue la voie la plus prometteuse pour donner à la famille innue la place qui lui revient (Guay et Grammond, 2012).

Issue du mode de vie nomade pratiqué depuis longtemps par les Innus, la famille élargie demeure d'actualité pour ceux-ci dans le contexte contemporain. C'est du moins ce qui ressort du témoignage que nous ont livré plusieurs membres de la communauté innue d'Uashat mak Mani-Utenam. Pour eux, la famille élargie constitue un véhicule de transmission de la culture et des valeurs et son importance dans l'organisation sociale se traduit concrètement, dans plusieurs cas, par des liens de cohabitation. En fait, on peut considérer la famille innue comme un ensemble de cercles concentriques au milieu duquel se trouve l'enfant, puis ses père et mère, ses parents rapprochés, puis l'ensemble de la communauté. Chaque cercle définit en quelque sorte un ensemble de responsabilités particulières envers l'enfant.

Les allochtones qui interviennent auprès des Innus ne peuvent plus se permettre d'ignorer la famille élargie. Les revendications des peuples autochtones pour une plus grande autonomie, notamment en matière de politique familiale et de protection de la jeunesse, peuvent être comprises comme une volonté de redonner à la famille élargie la place qui lui revient dans la société autochtone. Dans cette perspective, les initiatives récentes du gouvernement québécois représentent un pas dans la bonne direction. Le succès de leur mise en œuvre dépend toutefois, à notre avis, d'une bonne compréhension de la spécificité du concept de famille élargie. Le présent texte se veut une modeste contribution en ce sens. 


\section{BIBLIOGRAPHIE}

Assemblée des Premières Nations du Québec et du Labrador (APNQL) (2014). Protocole de recherche des Premières Nations au Québec et au Labrador, Wendake.

Basile, M.-J., R. Rock et J. Rock (1992). Montagnaise de parole: Eukuan ume ninan etentamat, Québec, Conseil des Atikamekw et des Montagnais.

Bertsch, M., et B. A. Bidgood (2010). «Why is adoption like a First Nations feast?: Lax Kw'alaam indigenizing adoptions in child welfare », First Peoples Child \& Family Review, vol. 5, no 1, p. 95105.

Boucher, N. (2005). La transmission intergénérationnelle des savoirs dans la communauté innue de Mashteuiatsh : les savoir-faire et les savoir-être au cœur des relations entre les Pekuakamiulnuats, mémoire de maîtrise inédit, Université Laval, Québec.

Brant Castellano, M. (2002). Tendances familiales autochtones. Les familles élargies, les familles nucléaires, les familles du cœur, Ottawa, Institut Vanier de la famille.

Breton, A., S. Dufour et C. Lavergne (2012). «Les enfants autochtones en protection de la jeunesse au Québec : leur réalité comparée à celle des autres enfants », Criminologie, vol. 45, $\mathrm{n}^{\circ} 2$, p. 157 185.

Brunschwig, A. (2015). Perception Atikamekw du phénomène de la grossesse adolescente. Analyse basée sur les récits de vies de douze femmes et deux hommes Atikamekw vivant à La Tuque (Région de la Mauricie, QC, Canada), mémoire de maîtrise inédit, Université Lumière Lyon 2, Lyon.

Charest, P. (2006). « Les Montagnais d'autrefois, les Innus d'aujourd'hui », Cap-aux-Diamants, n 85, p. 10-15.

Charest, P. (2001). "Les Montagnais ou Innus », dans G. Duhaime (dir.), Le Nord, habitants et mutations, Québec, Presses de l'Université Laval.

Delisle-L'Heureux, C. (2018). Les voix politiques des femmes innues face à l'exploitation minière, Québec, Presses de l'Université du Québec.

Di Tommasso, L., et S. de Finney (2015). «A discussion paper on Indigenous custom adoption. Part 2: Honoring our caretaking traditions », First Peoples Child \& Family Review, vol. 10, n 1, p. 19-38.

Gentelet, K., A. Bissonnette et G. Rocher (2005). La sédentarisation : effets et suites chez des Innus et des Atikamekw, Montréal, Les Éditions Thémis.

Grammond, S., et C. Guay (2016). "Comprendre la normativité innue en matière d'adoption et de "garde" coutumière », Revue de droit de McGill, vol. 61, n 4, p. 885-906. 
Guay, C. (2015). "Les familles autochtones : des réalités sociohistoriques et contemporaines aux pratiques éducatives singulières », Intervention, $\mathrm{n}^{\circ} 141$, p. 17-27.

Guay, C., et S. Grammond (2012). «Les effets des régimes de protection de la jeunesse sur les familles autochtones », Nouvelles pratiques sociales, vol. 25, n 1, p. 67-83.

Irvine, K. (2009). Soutien pour parents autochtones. Enseignements pour l'avenir, Prince George, Centre de collaboration nationale de la santé autochtone, UBC.

Kline, M. (1992). "Child Welfare Law, "best interests of the child" ideology, and First Nations », Osgoode Hall Law Journal, vol. 30, p. 375-425.

Lacasse, J.-P. (2004). Innu Tipenitamun. Les Innus et le territoire, Québec, Septentrion.

Lanari, R. E. (1973). L'adoption chez les Amérindiens Montagnais-Naskapis, North West River, Labrador, thèse de doctorat, Université Memorial de Terre-Neuve, Saint-Jean.

Lavallée, C. (2013). “L'adoption coutumière autochtone et l'adoption légale québécoise : vers l'émergence d'une interface entre les deux cultures ? ». Dans G. Otis (dir.), L'adoption coutumière autochtone et les défis du pluralisme juridique, Québec, Presses de l'Université Laval, p. 35-71.

Lindstrom, G., P. Choate, L. Bastien, A. Weasel Traveller, S. Breaker, C. Breaker, W. Good Striker et E. Good Striker (2016). Nistawatsimin : Exploring First Nations parenting. A literature review and expert consultation with Blackfoot Elders, Calgary, Mount Royal University.

Mailhot, J. (1999). Au pays des Innus. Les gens de Sheshashit, Montréal, Recherches amérindiennes au Québec et José Mailhot.

Muir, N., et Y. Bohr (2014). "Contemporary practice of traditional Aborignal child rearing: A review », First Peoples Child \& Family Review, vol. 9, n 1, p. 66-79. 